\title{
Deficit in Memory Is Associated with Paradoxical Regulation of Magnesium and Manganese in Isolated Male Rats
}

\author{
Hamidreza Famitafreshi ${ }^{a} \quad$ Morteza Karimian $^{b}$ \\ ${ }^{a}$ Department of Physiology, Tehran University of Medical Science-International Campus, Tehran, Iran; \\ ${ }^{b}$ Department of Physiology, Tehran University of Medical Science, Tehran, Iran
}

\section{Keywords}

Magnesium · Manganese · Glutathione · Isolation •

Memory · Y-maze

\begin{abstract}
Background: Magnesium (Mg) and manganese (Mn) play an important role in brain development and glutathione, the master antioxidant in the brain, is necessary for the cognitive function. This study is aimed at determining the effect of magnesium and manganese concentrations in hippocampus and prefrontal cortex on the glutathione function. The reduction in glutathione leads to memory impairment in Y-maze in the isolation period. Methods: In this study, 16 male Sprague-Dawley rats were randomly divided into 2 groups: social and isolation. Based on previous studies, the isolation period was considered as 14 days, plus 1 week for acclimatization. On day 15, after assessing the memory with Y-maze, rats were examined for glutathione in serum, hippocampus, and prefrontal cortex. Also, serum, hippocampus, and prefrontal cortex were obtained for assessing $\mathrm{Mn}$ and Mg. Results: The memory in Y-maze was impaired in isolated rats. Also, glutathione in hippocampus and prefrontal cortex was reduced in isolated rats versus socialized rats. In
\end{abstract}

\section{KARGER}

(C) 2018 S. Karger AG, Basel

E-Mail karger@karger.com

www.karger.com/aon serum assay, glutathione reduced in socialized rats versus isolated rats. Mg was reduced in isolated rats versus socialized rats in serum assay. Paradoxically, Mn was increased in isolated rats versus socialized rats in serum assay. In tissue assay, Mg in hippocampus increased in socialized rats versus isolated rats. Paradoxically, in prefrontal cortex Mg increased in isolated rats versus socialized rats. $\mathrm{Mn}$ in hippocampus showed increased concentration in socialized rats versus isolated rats. Paradoxically, Mn showed reduced concentration in prefrontal cortex in socialized rats versus isolated rats. Conclusion: Manganese and magnesium are necessary elements for maintaining enough level of cognition in the isolation period. Also, glutathione plays an important role.

(c) 2018 S. Karger AG, Basel

\section{Introduction}

Manganese (Mn) is an essential element for development, metabolism, and the antioxidant system that is more concentrated in some brain subregions such as the hippocampus [1]. Around brain fetal development, $\mathrm{Mn}$ is mostly concentrated in CA3 and dentate gyrus of the hippocampus. During adulthood, $\mathrm{Mn}$ is supplied to the brain 
via both the blood-brain and the blood-cerebrospinal fluid barriers [2]. Increased Mn level in the brain is associated with neurological disorders such as Parkinson disease $[3,4] . \mathrm{Mn}$ in brain is usually deposited in Striatum and Globus Pallidus [5]. It has been shown that Mn deficiency also causes working memory deficits [6].

Magnesium ( $\mathrm{Mg}$ ) in brain has specific functions. It is involved in modulating the voltage-dependent block of NMDAR, controlling their opening during coincidence detection that is critical for synaptic plasticity. Mg concentration is higher in the cerebrospinal fluid than in the plasma fluid [2]. Also, magnesium has been implicated based on previous studies to be important for proper brain function such as overcoming stress, anxiety, depression, migraine headache, and memory decline [7].

Reduced glutathione (GSH) is necessary for brain functions such as memory [8]. In previous literature, much attention was given based on experimental findings to the importance of antioxidant defense in brain disease. For example, glutathione has been implicated to be important in some psychiatric diseases such as schizophrenia and depression [9].

Contribution of prefrontal cortex and hippocampus are necessary for the proper functioning of memory. Contribution of prefrontal cortex and hippocampus is not necessary for all stages of memory formation [10]. Overall prefrontal cortex has a complementary function on memory formation. In some memories, encoding of prefrontal cortex is not mandatory [11].

In this study, the effect of environmental modulation such as social isolation on memory performance and the associated decline in antioxidant defense are investigated. Also, Mg and Mn balance are studied.

\section{Methods and Materials}

Animal care: Rats (about 9 weeks old) were housed individually in standard, small polycarbonate cage $(27 \times 15 \times 21)$ or together in large $(42 \times 15 \times 21)$ polycarbonate cage with ad libitum access to food and water. Cages were maintained in a temperature-controlled $\left(22 \pm 1^{\circ} \mathrm{C}\right)$ and humidity-controlled (40-70\%) vivarium with a $12 \mathrm{~h}$ light/dark cycle (lights on at 7:00 a.m.). Behavioral testing took place during the light cycle (specifically, from 11:00 a.m. to 3:00 p.m.) in the experimental room. All rats were habituated to the Tehran University of Medical Science vivarium for at least 7 days before the start of experiment. All experimental protocols were in accordance with the Animal Ethics Committee of Tehran University of Medical Sciences. Animals: In this study male, Sprague-Dawley rats weighing about 200-250 g were used. In each group, 8 rats were used; 8 rats were used for inducing social state.

Experimental procedure and preparation of blood and tissue: After 14 days, rats' memories in both groups were studied by
Y-maze. Then rats were sedated with xylazine $(10 \mathrm{mg} / \mathrm{kg})$ and anesthetized with ketamine $(100 \mathrm{mg} / \mathrm{kg})$. Blood was taken from the heart, and serum was prepared. The brain was removed from the skull and immediately frozen in liquid nitrogen. For the preparation of homogenized tissue, the prefrontal cortex and hippocampus were isolated from the brain and homogenized with buffer phosphate after weighing with digital balance (1/10).

Assessing magnesium and manganese in tissue and serum: After processing of serum and tissue as mentioned, the sample was diluted with nitric acid $65 \%$ and perchloric acid $65 \%$ to a total volume of $5 \mathrm{~mL}$. Then, $\mathrm{Mn}$ and $\mathrm{Mg}$ were assessed with an atomic spectrophotometer (Vivian). The wavelength was corrected with a standard curve and final concentrations were obtained [12].

Glutathione measurement: The prefrontal cortex and hippocampus were dissected from the brain and homogenized with phosphate buffer $(1 / 10)$. Then the reduced glutathione was measured based on Ellman reaction. Tris buffer, DTNB (5, 5' -dithiolbis-[2-nitrobenzoic acid]), and methanol were mixed together and $100 \mathrm{~mL}$ of homogenized tissue and serum were added to the above mixture. The maximum absorbance of the final solution was assessed with a spectrophotometer at a wavelength of $412 \mathrm{~nm}$ [8].

Y-maze: Short-term spatial memory was assessed with Y-maze apparatus. Briefly, Y-maze apparatus has 3 arms that cross each other at $120^{\circ}$. Rats were subjected to explore the new arms, and the least mistake in exploring the last explored arm determined. The least frequency in exploring the last explored arm is an indicator of better memory performance. The frequency of exploration of 3 consecutive new arms (A, B, and C) was considered the actual alternation. The scores were calculated as follows: (actual alternation/maximal alternation-2) $\times 100$. Also, the number of entries was recorded [13].

Statistics: Independent two-tailed sample $t$ test was performed for comparison of the 2 groups of experiments. SPSS version 22 was used for statistical analysis. Data were represented as mean \pm SEM. $p<0.05$ was considered significant.

\section{Result}

Manganese in hippocampus and prefrontal cortex tissues: In socially isolated rats, $\mathrm{Mn}$ in hippocampus was reduced compared to that of social rats $(0.8125 \pm$ 0.2056 vs. $0.7625 \pm 0.03750$ ppm, $p=0.0044)$. Paradoxically, in prefrontal cortex Mn was increased in socially isolated rats compared to that of social rats $(0.75 \pm$ 0.01667 vs. $0.8875 \pm 0.02394 \mathrm{ppm}, p=0.0013$; Figure 1 ).

Magnesium in hippocampus and prefrontal cortex tissues: In socially isolated rats, $\mathrm{Mg}$ in hippocampus was reduced compared to that of social rats $(53.88333 \pm$ 1.89 vs. $43.05 \pm 7.75 \mathrm{ppm}, p=0.0434)$. Paradoxically, in prefrontal cortex, $\mathrm{Mg}$ was increased in socially isolated rats compared to that of social rats $(39.26667 \pm 3.299$ vs. $53.7 \pm 3.947 \mathrm{ppm}, p=0.0260$; Figure 2).

Manganese and magnesium in serum: In socially isolated rats, $\mathrm{Mg}$ in serum showed reduced concentration 


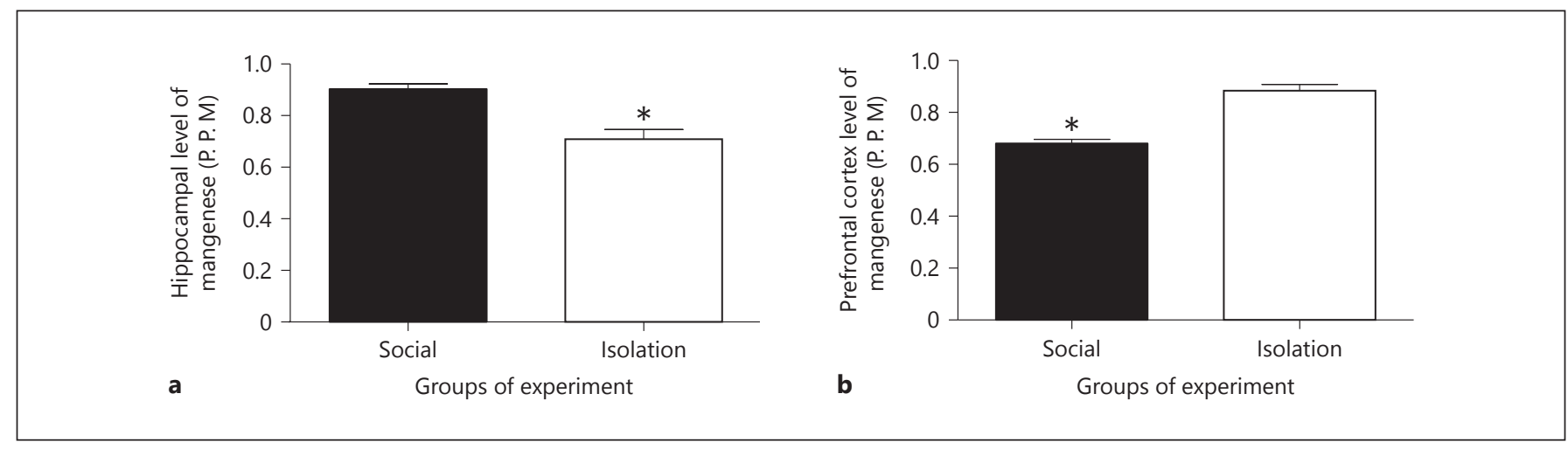

Fig. 1. The above figures shows concentrations of manganese in hippocampus (a) and prefrontal cortex (b) as measured by spectrophotometer after preparation for analysis. The wavelength was corrected with a standard curve for obtaining concentration. Data were represented as mean \pm SEM. A significant difference was considered for $p<0.05$.

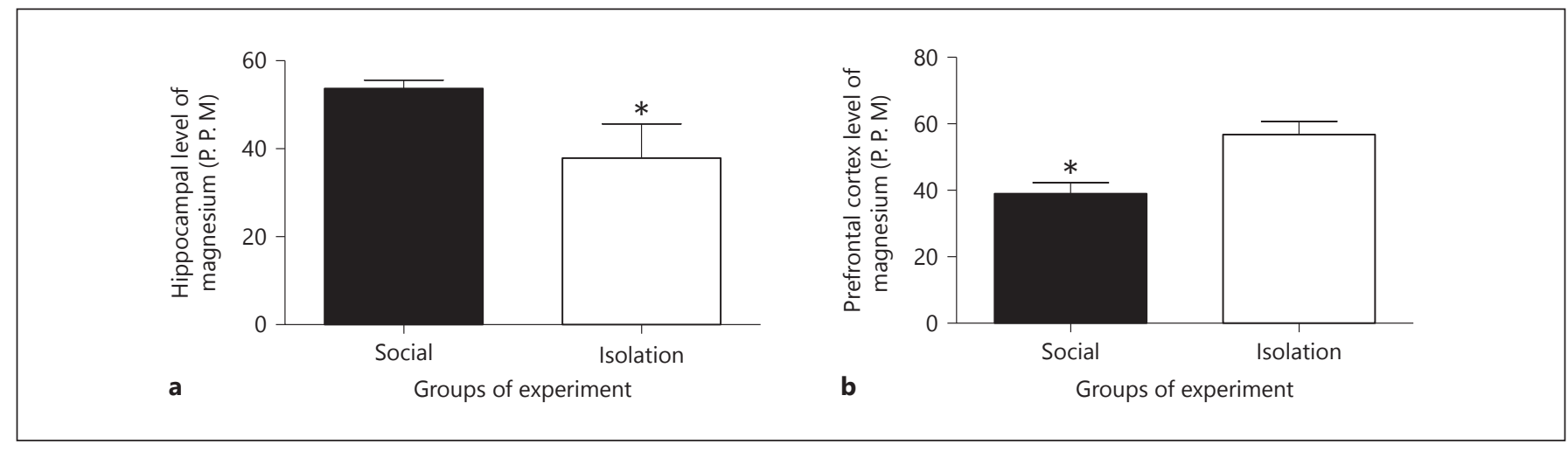

Fig. 2. The above figures shows concentrations of magnesium in hippocampus (a) and prefrontal cortex (b) as measured by spectrophotometer after preparation for analysis. The wavelength was

compared to that of social rats $(46.61667 \pm 6.699$ vs. $39.63333 \pm 2.110 \mathrm{ppm}, p=0.0364)$. Paradoxically, in social rats $\mathrm{Mn}$ showed reduced concentration compared to that of socially isolated rats $(0.5575 \pm 0.0075$ vs. $0.6125 \pm$ $0.050 \mathrm{ppm}, p=0.0286$; Figure 3).

Glutathione in hippocampus and prefrontal cortex: In socially isolated rats, glutathione in hippocampus tissue showed reduced concentration compared to that of social rats $(3.465 \pm 0.145$ vs. $1.975 \pm 0.0250 \mu \mathrm{mol} / \mathrm{L}$, $p=0.0096)$. Similarly, in socially isolated rats glutathione in prefrontal cortex showed reduced concentration compared to socially isolated rats $(2.473333 \pm 0.1819$ vs. $1.655 \pm 0.2150 \mu \mathrm{mol} / \mathrm{L}, p=0.0318$; Figure 4 ).

Glutathione in serum: In socially isolated rats, glutathione in serum was increased compared to that of social rats $(0.8690 \pm 0.1190$ vs. $3.150 \pm 0.4852 \mu \mathrm{mol} / \mathrm{L}, p=$ 0.0346 ; Figure 5).

Paradoxical Regulation of Magnesium and Manganese corrected with a standard curve for obtaining concentration. Data were represented as mean \pm SEM. A significant difference was considered for $p<0.05$.

Food intake: In socially isolated rats, food intake was reduced compared to that of social rats $(24.32 \pm 1.965$ vs. $17.46 \pm 0.5472 \mathrm{~g}, p=0.0246$; Figure 6).

Y-maze: In assessing short-term working memory, social rats got better scores than socially isolated rats (84.33 \pm vs. $5.04151 .2 \pm 6.677 \%, p=0.0316)$. Also, the number of entries was higher in socially isolated rats, which might be indicative of forced efforts in choosing the right entrance ( $8 \pm 0.9989$ vs. $15 \pm 2.009[n], p=0.0105$; Figure 7).

\section{Discussion}

In this study, it was shown that in isolation period (1) $\mathrm{Mn}$ and $\mathrm{Mg}$ are paradoxically regulated in serum, in hippocampus and prefrontal cortex, (2) glutathione is re- 


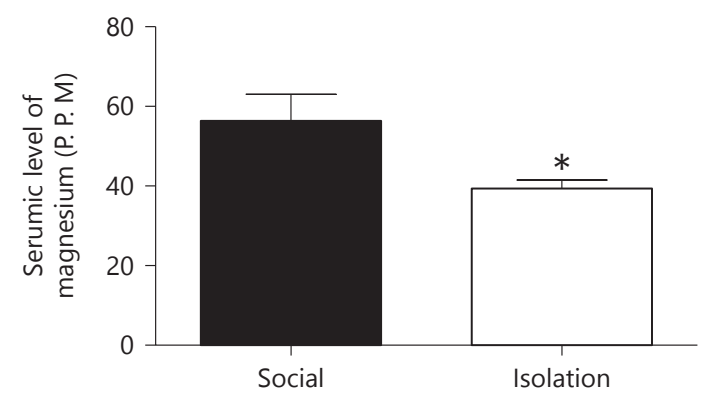

a

Groups of experiment
Fig. 3. The above figures shows concentrations of manganese and magnesium in serum (a, b) as measured by spectrophotometer after preparation for analysis. The wavelength was corrected with a

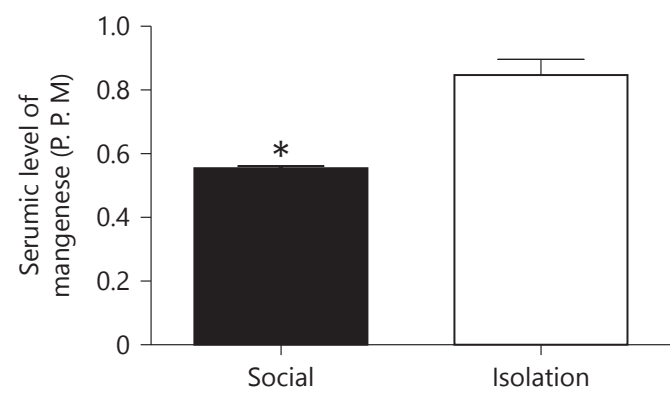

b

Groups of experiment standard curve for obtaining concentration. Data were represented as mean \pm SEM. A significant difference was considered for $p<$ 0.05 .

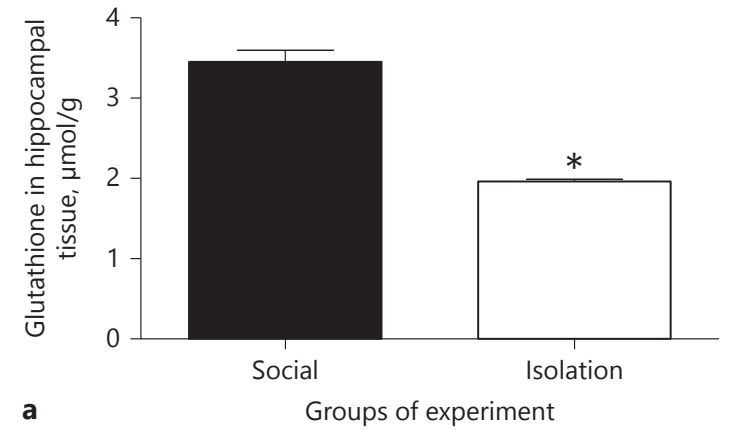

Fig. 4. The above figures shows concentrations of glutathione in hippocampus (a) and prefrontal cortex (b) as measured by spectrophotometer after preparation for analysis. The wavelength was

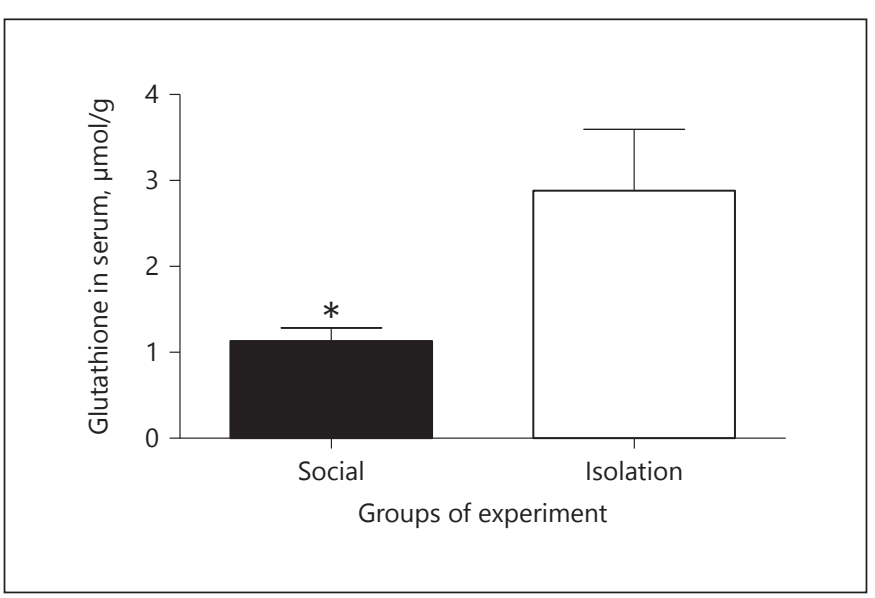

Fig. 5. The above figures shows concentrations of glutathione in serum as measured by spectrophotometer after preparation for analysis. The wavelength was corrected with a standard curve for obtaining concentration. Data were represented as mean \pm SEM. A significant difference was considered for $p<0.05$. corrected with a standard curve for obtaining concentration. Data were represented as mean \pm SEM. A significant difference was considered for $p<0.05$.

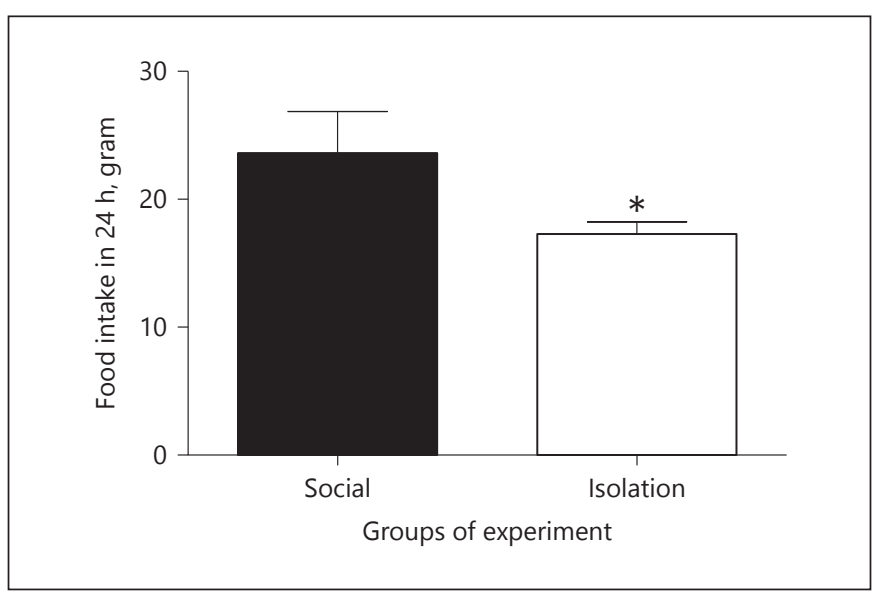

Fig. 6. The above figures shows food intake per day. Data were represented as mean \pm SEM. A significant difference was considered for $p<0.05$. 

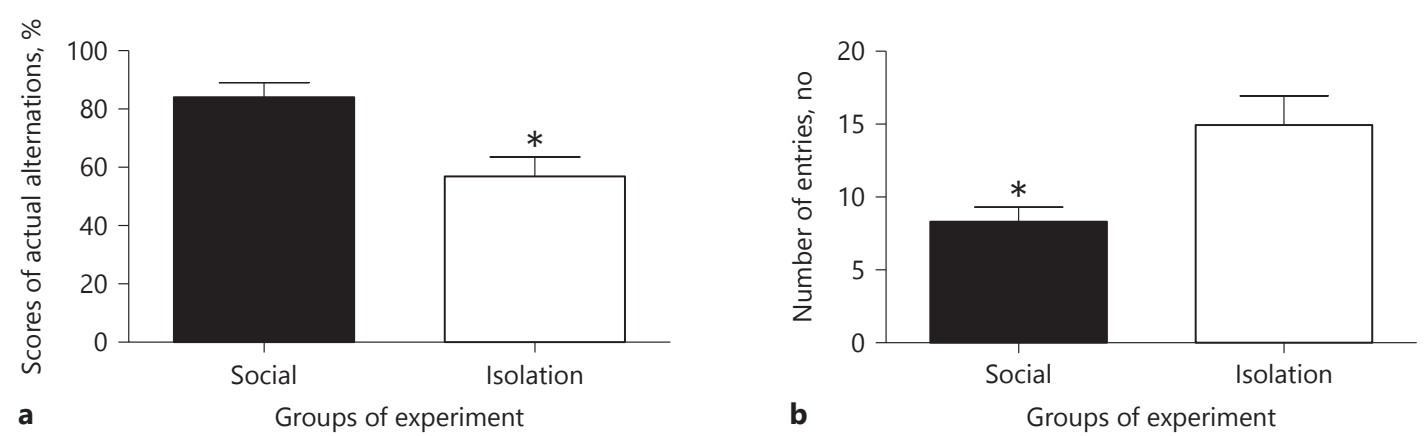

Fig. 7. The above figures show memory function in Y-maze. (a) Shows the percentage of right choices in an experiment that is measure spatial working short term memory. (b) Shows the a number of entries to each three arms overall. The number of entries may

duced in the prefrontal cortex and hippocampus, and (3) memory performance is impaired in Y-maze.

The concentration of $\mathrm{Mg}$ in both the extracellular and intracellular fluids is regulated by different mechanisms [14]. Renal regulation of $\mathrm{Mg}$ concentration is an important mechanism for avoiding the accumulation of $\mathrm{Mg}$ in the body. Mg in this study accumulated in prefrontal cortex in isolated rats.

Also, Mn transport is regulated by different mechanisms. Based on the experiment, brain has a great ability to concentrate $\mathrm{Mn}$ [15]. Mn in this study accumulated in the prefrontal cortex in isolated rats.

Glutathione is present in all types of brain tissues as an antioxidant. It is mostly found in brain cortex, corpus striatum, and the glia [16]. The reason for the higher presence of glutathione is related to high level of cysteine in glial cells that serve as a precursor for glutathione synthesis [17]. In recent times, it has got great attention as an agent that is important for brain function and reduction of burden of neurologic diseases. For example, in one study glutathione showed a beneficial effect for Parkinson disease [18] and in another glutathione treatment reduced the burden of Alzheimer disease [19]. Also, glutathione in several studies has been shown to be important for memory recovery and improvement that is assessed by Y-maze [8]. The mechanisms improve the memory and other symptoms of brain disease that are poorly understood. It should be noted that in our previous experiment because of some difficulties we failed to show that isolation decreases the memory in Y-maze [20].

In this study, we observed that $\mathrm{Mn}$ and $\mathrm{Mg}$ were paradoxically regulated in serum. Mn was increased in the isolation period but $\mathrm{Mg}$ decreased. This may reflect

Paradoxical Regulation of Magnesium and Manganese 


\section{Disclosure Statement}

The authors have no conflicts of interest to declare.

\section{Funding Source}

This work has been completed by personal funding.

\section{Authorship Contribution}

This work was carried out in collaboration between 2 authors. Author H.F. designed the study, wrote the protocol, performed experiments and statistical analysis with assistance from author M.K. Author H.F. managed the literature searches, wrote the first and final drafts of the manuscript and edited the final manuscript. All authors read and approved the final manuscript.

\section{References}

1 Takeda A, Ishiwatari S, Okada S: Manganese uptake into rat brain during development and aging. J Neurosci Res 1999;56:93-98.

2 Bradbury MW, Crowder J, Desai S, Reynolds JM, Reynolds M, Saunders NR: Electrolytes and water in the brain and cerebrospinal fluid of the foetal sheep and guinea-pig. J Physiol 1972;227:591-610.

3 Ratner MH, Fitzgerald E: Understanding of the role of manganese in parkinsonism and Parkinson disease. Neurology 2017;88:338339.

4 Kwakye GF, Paoliello M, Mukhopadhyay S, Bowman AB, Aschner M: Manganese-induced parkinsonism and Parkinson's disease: shared and distinguishable features. Int J Environ Res Public Health 2015;12:75197540 .

5 Reaney SH, Bench G, Smith DR: Brain accumulation and toxicity of Mn (II) and Mn (III) exposures. Toxicol Sci 2006;93:114-124.

6 Chang Y, Lee JJ, Seo JH, Song HJ, Kim JH, Bae SJ, Ahn JH, Park SJ, Jeong KS, Kwon YJ, Kim $\mathrm{SH}$, Kim Y: Altered working memory process in the manganese-exposed brain. Neuroimage 2010;53:1279-1285.

7 Eby GA, Eby KL: Rapid recovery from major depression using magnesium treatment. Medical Hypotheses 2006;67:362-370.

8 Dean O, Bush AI, Berk M, Copolov DL, van den Buuse M: Glutathione depletion in the brain disrupts short-term spatial memory in the Y-maze in rats and mice. Behav Brain Res 2009; 198:258-262.
9 Pandya CD, Howell KR, Pillai A: Antioxidants as potential therapeutics for neuropsychiatric disorders. Neuropsychopharmacol Biol Psychiatry 2013;46:214-223.

10 Preston AR, Eichenbaum H: Interplay of hippocampus and prefrontal cortex in memory. Curr Biol 2013;23:R764-R773.

11 Frankland PW, Bontempi B, Talton LE, Kaczmarek L, Silva AJ: The involvement of the anterior cingulate cortex in remote contextual fear memory. Science 2004;304:881-883.

12 Yang MS, Wong MH: Changes in $\mathrm{Ca}, \mathrm{Cu}, \mathrm{Fe}$, $\mathrm{Mg}$, and $\mathrm{Zn}$ contents in mouse brain tissues after prolonged oral ingestion of brick tea liquor containing a high level of Al. Biol Trace Elem Res 2001;80:67-76.

13 Mandillo S, Tucci V, Hölter SM, Meziane H, Banchaabouchi MA, Kallnik M, Lad HV, Nolan PM, Ouagazzal AM, Coghill EL, Gale K, Golini E, Jacquot S, Krezel W, Parker A, Riet F, Schneider I, Marazziti D, Auwerx J, Brown SD, Chambon P, Rosenthal N, Tocchini-Valentini G, Wurst W: Reliability, robustness, and reproducibility in mouse behavioral phenotyping: a cross-laboratory study. Physiol Genomics 2008;34:243-255.

14 Cefaratti C, Romani AM: Functional characterization of two distinct $\mathrm{Mg} 2+$ extrusion mechanisms in cardiac sarcolemmal vesicles. Mol Cell Biochem 2007;303:63-72.

15 Sotogaku N, Oku N, Takeda A: Manganese concentration in mouse brain after intravenous injection. J Neurosci Res 2000;6:350356.
16 Philbert MA, Beiswanger CM, Waters DK, Reuhl KR, Lowndes H: Cellular and regional distribution of reduced glutathione in the nervous system of the rat: histochemical localization by mercury orange and o-phthaldialdehyde-induced histofluorescence. Toxicol Appl Pharmacol 1991;107:215-227.

17 Keelan J, Allen NJ, Antcliffe D, Pal S, Duchen MR: Quantitative imaging of glutathione in hippocampal neurons and glia in culture using monochlorobimane. J Neurosci Res 2001; 66:873-884.

18 Michels L, Schulte-Vels T, Schick M, O'Gorman RL, Zeffiro T, Hasler G, MuellerPfeiffer C: Prefrontal GABA and glutathione imbalance in posttraumatic stress disorder: preliminary findings. Psychiatry Res 2014; 224:288-295.

19 Pocernich CB, Butterfield DA: Elevation of glutathione as a therapeutic strategy in Alzheimer disease. Biochim Biophys Acta 2012; 1822:625-630.

20 Famitafreshi H, Karimian M, Fanaei H, Attari F, Fatima S: Social isolation is associated with reduced neurogenesis, impaired spatial working memory performance and altered anxiety levels in male rats. Open Access Anim Physiol 2015;7:87-95.

21 Yavuz Y, Mollaoglu H, Yürümez Y, Ucok K, Duran L, Tünay K, Akgün L: Therapeutic effect of magnesium sulphate on carbon monoxide toxicity-mediated brain lipid peroxidation. Eur Rev Med Pharmacol Sci 2013; 17(suppl 1):28-33. 